\title{
COMPORTAMIENTO ARBÓREO DEL SAPO DE CAÑA MESOAMERICANO (RHINELLA HORRIBILIS) EN COQUUIMATLÁN, COLIMA, MÉXICO
}

\section{ARBOREAL BEHAVIOR OF THE MESOAMERICAN CANE TOAD (RHINELLA HORRIBILIS) AT COQUUIMATLAN, COLIMA, MEXICO}

\section{DAMIAN BERRA-ALANIS}

Instituto de Investigaciones Sobre los Recursos Naturales, Universidad Michoacana de San Nicolás de Hidalgo, San Juanito Itzicuaro, s/n Col. Nva. Esperanza 58337, Morelia, Michoacán, México.

Preparatoria "Rector Melchor Días Rubio" A.C., Álvaro Obregón Sur No. 403 Col. Centro C.P 61420 Tuxpan, Michoacán, México.

Correspondence: damianberraal@gmail.com

Abstract.- I report an observation of the anuran Rhinella horribilis (Mesoamerican cane toad) from Mexico perched on a tree branch $194 \mathrm{~cm}$ above the forest floor. The toad is suspected to have been foraging on ants that were observed walking on the branch, although a direct observation of predation by the toad was not observed. This report provides new information about the natural history of this species.

Keywords.- Arboreal behavior, climbing behavior, Rhinella marina, ants, tree.

Resumen.- Se reporta en México la observación del anuro Rhinella horribilis (sapo de caña mesoamericano) situado en la rama de un árbol de $194 \mathrm{~cm}$ de altura. Se sospecha que el sapo estaba forrajeando, ya que se observaron hormigas caminando en la rama, aunque no se observó al sapo directamente involucrado en la depredación. Este reporte provee nueva información sobre la historia natural de esta especie.

Palabras clave.- Comportamiento arbóreo, comportamiento de escalada, Rhinella marina, hormigas, árbol.

El sapo de caña mesoamericano (Rhinella horribilis), es un anuro de la familia Bufonidae, este junto al sapo de la caña (Rhinella marina) son considerados unos de los anfibios de mayor tamaño en América, de hábitos terrestres y comparte similitudes morfológicas, excepto por caracteres en la morfometria craneal (Acevedo et al. 2016). Es una especie nativa de América con amplia distribución que va desde el sur de Texas hasta la región de la Cordillera de los Andes (Ron \& Read 2012, Bonett et al. 2017). Presenta una dieta generalista, alimentándose de una gran variedad de invertebrados, vertebrados pequeños y en algunos casos de plantas (Botero-Trujillo 2006, Escudero \& Ortega 2009, Sampedro-Marín et al. 2011).

Hudson et al. (2016) reporta que en algunas regiones de Australia y Hawaii, donde ha sido introducido, $R$. marina trepa a superficies casi verticales (troncos de árboles, acantilados, cercas) durante actividades de alimentación o dispersión, durante el estudio realizaron pruebas de escalada en los sapos de caña con lo cual concluyeron que al menos en algunas poblaciones lo sapos son expertos escaladores. En esta contribución, informo cuál es, según mi conocimiento, el primer caso documentado de comportamiento escansorial en la especie estrechamente relacionada $R$. horribilis.

En la localidad de El Chical $\left(19.229007^{\circ},-103.849492^{\circ}\right.$, elev. 380 m WGS84) situada en el Municipio de Coquimatlán en el Estado de Colima, México, el día 20 de Julio del 2018, se observó durante un muestreo nocturno a la 01:23 horas un ejemplar adulto de $R$. horribilis sobre un árbol joven de mango (Mangifera $s p$.) (Figura 1) localizado cerca de un manantial con vegetación riparía y remanentes de bosque tropical seco. El animal posiblemente se encontraba forrajeando ya que se observaron hormigas negras de la familia Formicidae en las ramas del árbol, aunque no se observó al sapo directamente involucrado en la depredación. El ejemplar se encontró sobre una rama gruesa bifurcada a $343 \mathrm{~cm}$ desde la base del tronco (Figura 2). La rama estaba inclinada formando un ángulo de $46^{\circ}$ y con una distancia recta desde la horqueta al suelo de $194 \mathrm{~cm}$. La corteza del árbol presentaba surcos reticulados poco profundos y la base del árbol se encontraba a la orilla de un canal del riego. 


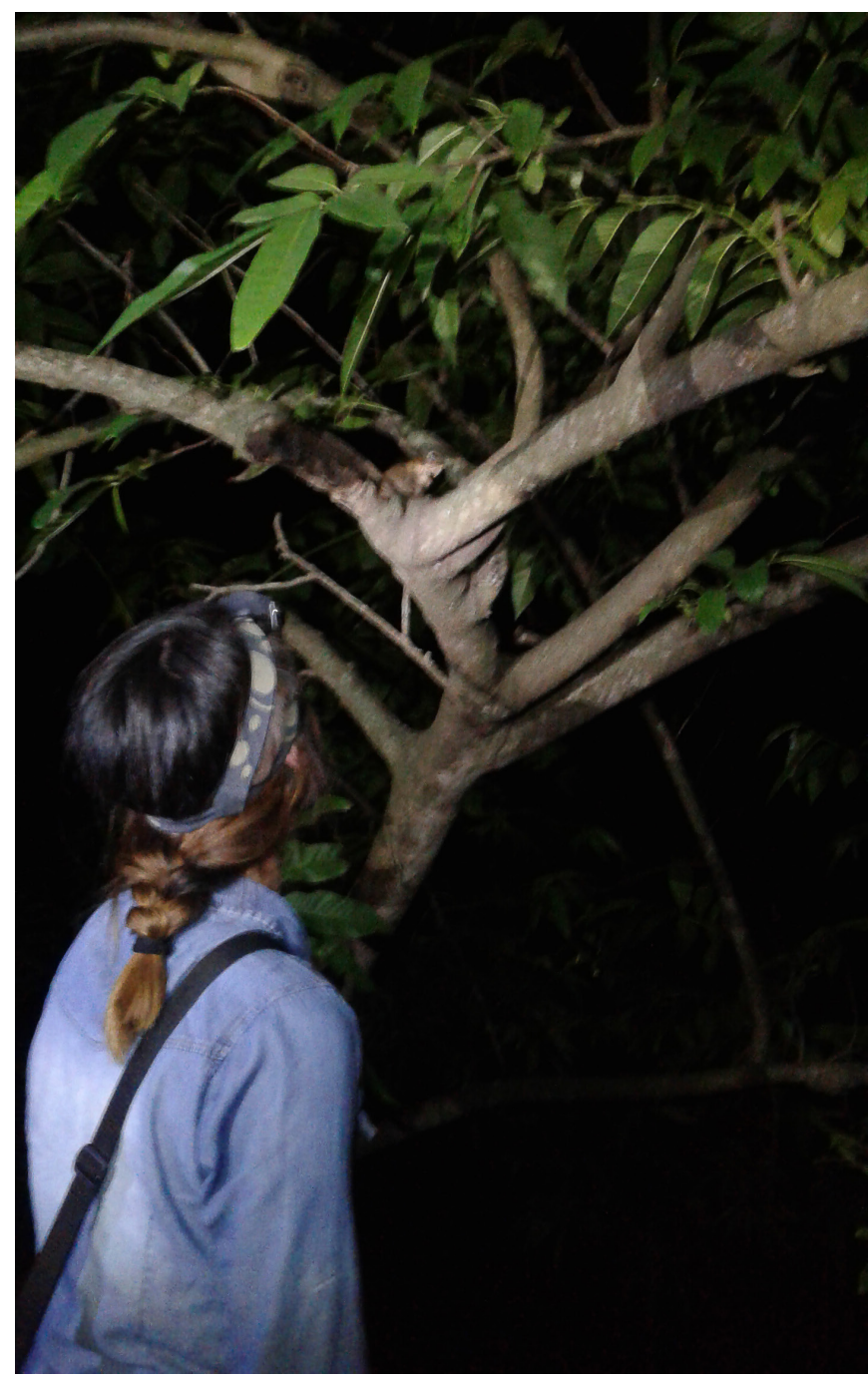

Figure 1. Position Height of R. horribilis on the mango tree (Mangifera sp.) over the ground. (Photography of Damian Berra Alanis).

Figura 1. Altura de la posición de R. horribilis en el árbol de mango (Mangifera sp.) con respecto al suelo. (Fotografia de Damian Berra Alanis).

En concordancia con el estudio realizado por Hudson et al. (2016), podemos deducir que la especie $R$. horribilis es capaz de trepar por superficies inclinadas, como lo demuestra la evidencia registrada.

\section{LITERATURA CITADA}

Acevedo, A.A., M. Lampo, \& R. Cipriani. 2016. The cane or marine toad, Rhinella marina (Anura, Bufonidae): two genetically and morphologically distinct species. Zootaxa, 4103(6):574-586.

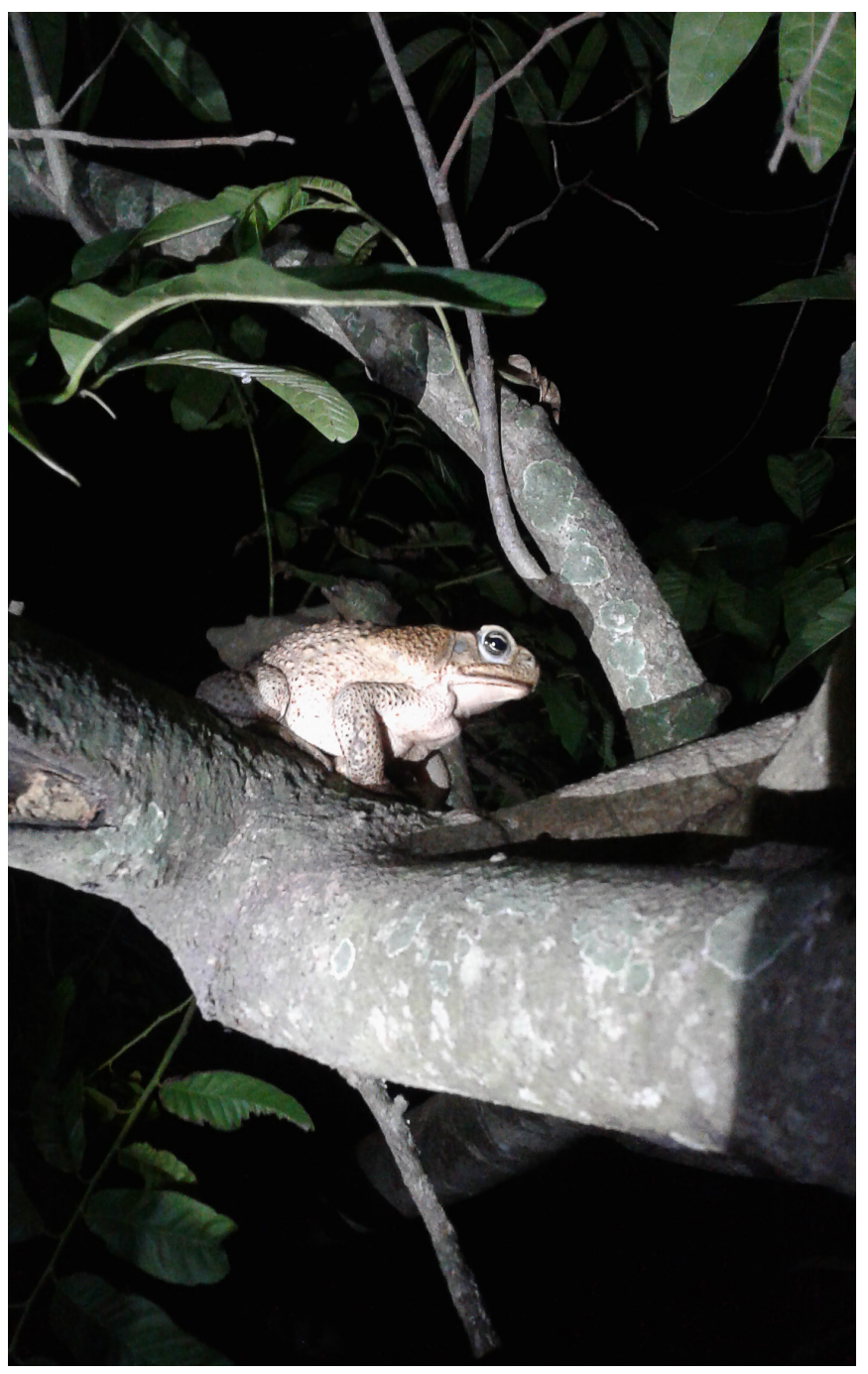

Figure 1. Position of R. horribilis on the mango tree (Mangifera sp.). (Photography of Damian Berra Alanis).

Figura 1. Posición de R. horribilis en el árbol de mango (Mangifera sp.). (Fotografia de Damian Berra Alanis).

Botero-Trujillo, R. 2006. Anuran predators of scorpions: Bufo marinus (Linnaeus, 1758) (Anura: Bufonidae), first known natural enemy of Tityus nematochirus Mello-Leitão, 1940 (Scorpiones: Buthidae). Revista Ibérica de Aracnología, 13:199-202.

Bonett, R.M., J. Boundy, F.T. Burbrink, B.I. Crother, K. de Queiroz, D.R. Frost, \& K.L Krysko. 2017. Scientific and Standard English Names of Amphibians and Reptiles North of Mexico, with Comments Regarding Confidence in Our Understanding. 
Escudero, M.B. \& A.M.J. Ortega, 2009. Dieta Entomofágica en Una Población de Rinella Marina en La Selva Pluvial Central, Chocó, Colombia. Revista Institucional Universidad Tecnológica Del Chocó Investigación Biodiversidad Y Desarrollo, 28(2).

Hudson, C.M., G.P. Brown \& R. Shine. 2016. Athletic anurans: the impact of morphology, ecology and evolution on climbing ability in invasive cane toads. Biological journal of the Linnean Society, 119(4):992-999.
Ron, S.R. \& M. Read. 2012. Rhinella horribilis. En Ron, S. R., J.M. Guayasamin, M.H. Yanez-Muñoz, A. Merino Viteri, D.A. Ortiz, \& D.A. Nicolalde. 2017. AmphibiaWebEcuador. Version 2017.0. Museo de Zoología, Pontificia Universidad Católica del Ecuador, 30 de mayo 2019.

Sampedro-Marín, A.C., Y.Y. Angulo Villalba, F.I. Arrieta Diaz \& D.M. Dominguez Atencia. 2011. Alimentación de Bufo marinus (Linnaeus, 1758) (Bufonidae: Anura), en una localidad de Sucre, Colombia. Caldasia, 33(2):495-505. 